\title{
Diabetes Care in Singapore
}

\author{
Goh Su-Yen, ${ }^{1}$ Lee Chung Horn, ${ }^{2}$ Bee Yong Mong ${ }^{1}$ \\ ${ }^{1}$ Singapore General Hospital, Singapore Health Services Pte Ltd \\ ${ }^{2}$ Lee Chung Horn Diabetes and Endocrinology Pte Ltd
}

\begin{abstract}
Singapore, like her ASEAN counterparts, faces a human and economic burden of chronic diseases such as diabetes. The Singapore diabetes care model features a mixed financing and mixed care delivery model, with an increasing focus on integration of care across traditional boundaries, through information technology, clinical engagement, robust clinical governance and financing schemes, and patient education and self-management.
\end{abstract}

Key words: health services needs and demands, delivery of healthcare, Singapore

\section{INTRODUCTION}

Excellent and efficient healthcare delivery must surely be the aim of any healthcare system around the world. As our Prime Minister Lee Hsien Loong expounded at the Universal Health Coverage Ministerial Meeting in February 2015, good health is fundamental to the happiness, fulfilment and dignity of every human being and good health is a public good. ${ }^{1}$

\section{Disease burden}

Diabetes (including type 1 diabetes, but predominantly type 2 diabetes) accounted for $10.4 \%$ of the total disease and injury burden in Singapore in 2010. ${ }^{2}$ Demographic epidemiological modelling places the estimate at $15 \%$ in 2050 , with a similar prevalence of obesity at $15.9 \%$; and that ethnic Malay and Indians will continue to bear a disproportionate burden compared with the Chinese majority. ${ }^{3}$

Between 2004 and 2010, there was a 4.6\% increase in total disease burden: $15.9 \%$ increase in disability burden and decline of $59.9 \%$ in premature mortality burden. However, both crude and age-standardized overall burden per head of population decreased by $3.3 \%$ and $11.6 \%$, respectively. Less than one-tenth $(6 \%)$ of the burden were from premature mortality. Eighty percent of the total diabetes burden resulted from diabetes per se (i.e. the experience of being diabetic regardless of complications), and the remainder due to complications (neuropathy, diabetic foot, peripheral vascular disease, nephropathy,

\section{e-ISSN 2308-118X}

Printed in the Philippines

Copyright (C) 2015 by the JAFES

Received: August 30, 2015. Accepted: October 15, 2015.

http://dx.doi.org/10.15605/jafes.030.02.08 amputation and others). Diabetes was the second most important cause in overall disease-adjusted life years (DALYs), and was ranked first in overall DALYs for women. It was the second leading cause of DALYs for men. Total diabetes burden increased to $14.2 \%$ when the increased risk of ischemic heart disease and stroke attributed to diabetes was included. ${ }^{2}$

Singapore also has a high burden of pre-diabetes (impaired fasting glucose and impaired glucose tolerance), with $14.4 \%$ of its population between the ages of 18 and 69 having pre-diabetes. Pre-diabetes is associated with an increased risk of developing diabetes mellitus. Diabetes was the 8th most common cause of hospitalization in 2013.

\section{Current structure and financing}

The Singapore Healthcare system is constantly evolving, as it adapts to more chronic diseases like diabetes. Compared with 50 years ago, as disease patterns and practices have changed, we are now dealing with more diseases of lifestyle and affluence, and less with communicable diseases and ailments of poverty and sanitation.

Our system is perhaps best described as a sometimes uneasy marriage between a privatized healthcare system and a single-payer model, effected through a mixed financial system. The twin philosophies are individual responsibility and affordable healthcare for all, and the devil is in the details. In a majority of cases, Singaporeans have to co-pay for each medical service, treatment and prescription drug that they utilize or receive.

Corresponding author: Goh Su-Yen, FRCP

Singapore General Hospital, Singapore Health Services Pte Ltd

20 College Road, Academia, Singapore

169856, Republic of Singapore

Tel. No.: +6563214654

Fax No.: +65 62273576

E-mail: goh.su.yen@singhealth.com.sg 


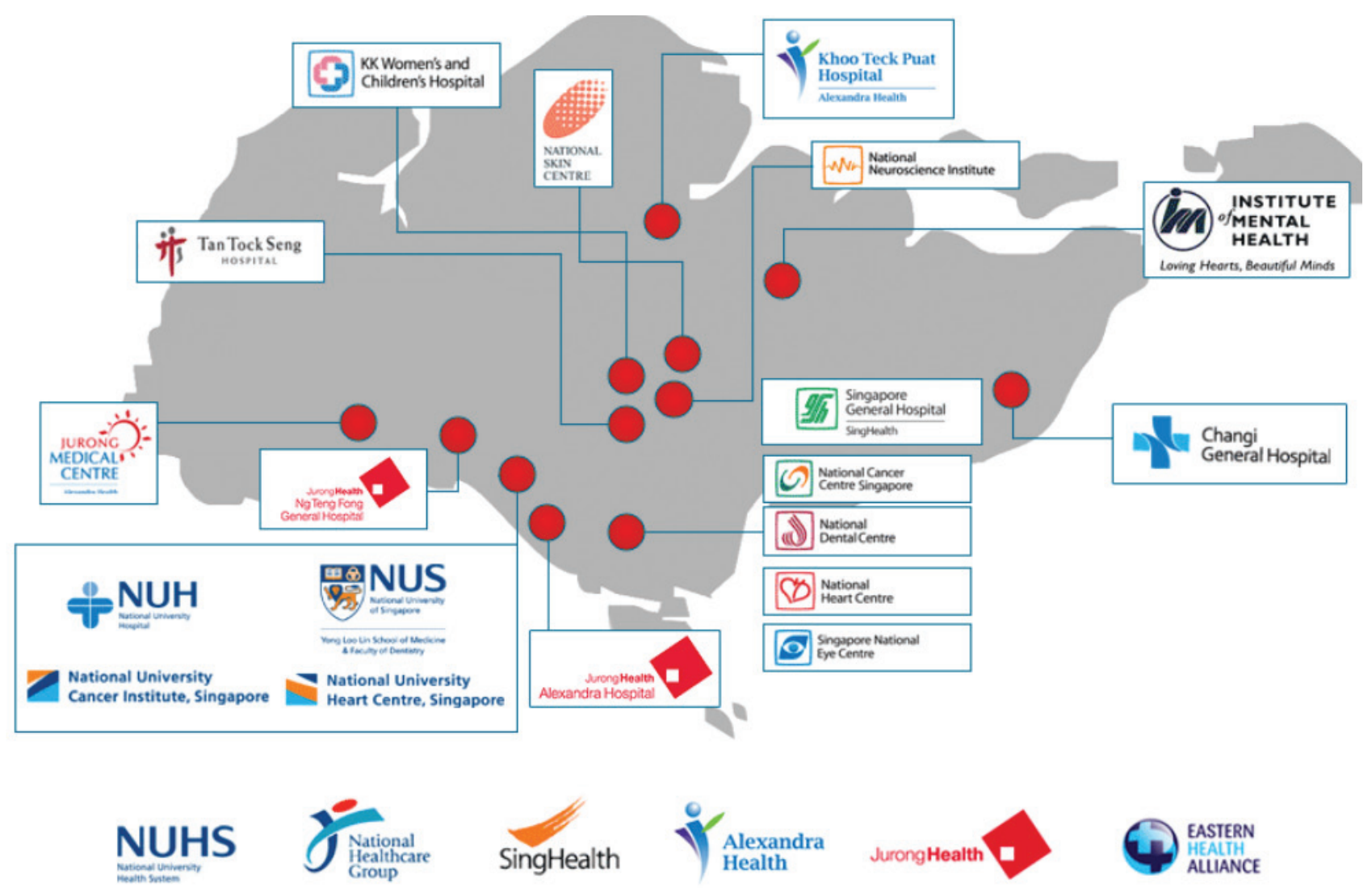

Figure 1. Restructured hospitals in Singapore.

The healthcare system is divided into two: the private and public (known as 'restructured') sector. The majority of hospital beds are owned by the restructured hospitals which continue to be owned by the government and supervised by the Ministry of Health, but function as nonprofit operating and accounting entities (Figure 1). Eighty percent of acute care is serviced by the restructured sector, but primary care is dominated by private providers. ${ }^{4}$ About 2000 private General Practitioners provide $80 \%$ of primary healthcare services, while public outpatient polyclinics provide $20 \%$ of the care.

Many community-based programs come under the purview of the Health Promotion Board (HPB), a World Health Organization Collaborating Centre for Health Promotion and Disease Prevention. The HPB conducts a myriad of programs addressing the public health concerns on diabetes, including a Pre-Diabetes Intervention Program offering three individual counselling sessions for 45 minutes at each time in community centers/clubs (CCs) or General Practice (GP) clinics. HPB also offers an interactive diet and activity tracker known as iDAT and blood glucose/blood pressure recording templates. As the lead agency spearheading health promotion and disease prevention, it has embarked on a multi-prong approach to target obesity, active living, smoking cessation, low-salt consumption, workplace health and community wellness.
Singapore has a compulsory medical savings scheme known as Medisave, launched in 1984. The underlying rationale is that the current workforce of active wageearners should save for their healthcare needs instead of relying on tax revenues from future generations and government handouts. Funds in Medisave accounts can also be used to pay for certain hospital-related expenses of immediate family members, in line with the principle of promoting the primacy of family in care-giving. Government subsidies are also based on price discrimination, allowing subsidies to be directed to poorer users in the lowest ward class. In the period of July 2014 to June 2015, the 50th percentile bill size for admissions related to uncomplicated diabetes in a C-class (lowest ward class, least add-ons) was in the range of S\$162 to 216 per day, with an average length of stay of 2.5 to 4.3 days. $^{4}$ In comparison, a hospitalization episode in a B1-class ward, which receives a lower level of subsidy, the 50th percentile bill size is $\mathbf{S} \$ 785$ per day. Items such as blood glucose monitoring and insulin pumps are entirely out-ofpocket expenses.

In the restructured/public healthcare system, patients receive subsidies for pharmacotherapeutic agents on a tiered basis depending on their paying status (maximum subsidy for the lowest payment class) and type of medication (maximum subsidy for drugs on the standard list such as generic glipizide and human insulin, and zero subsidy for SGLT2-inhibitor). 


\section{Vision of Future Healthcare Landscape} enabled by the Regional Health Systems (RHS)

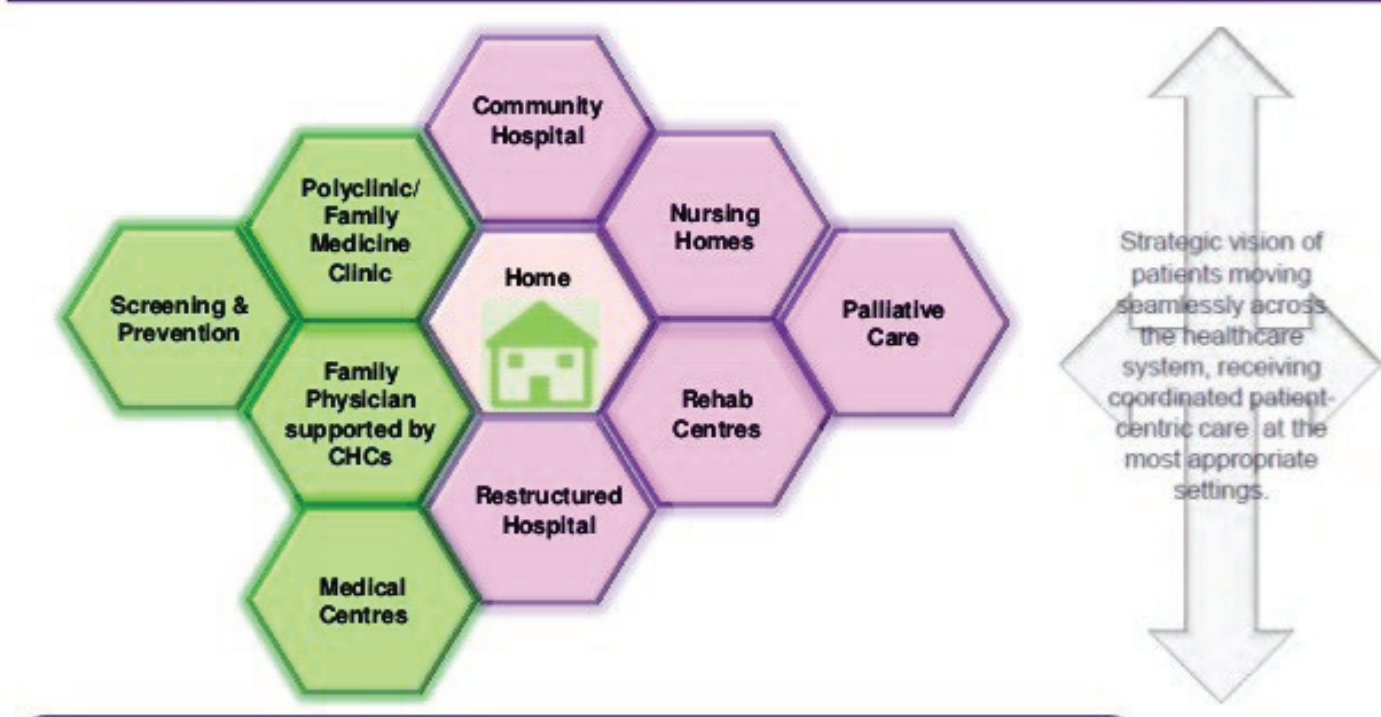

Organisation of the RHS, where Home is the central location for care, with primary care delivering person-centric care integrated with the rest of the care spectrum for both physical and mental healthcare.

Figure 2. Interface between general practice, community-based programs and secondary/tertiary services, under the Regional Health Systems.

The Chronic Disease Management Programme (CDMP) was first introduced in October 2006 for diabetes mellitus, hypertension, hyperlipidemia and stroke, permitting the use of a family member's or one's own Medisave (up to 10 accounts) of up to $\$ \$ 400$ per account per year. Each claim is subject to a $15 \%$ co-payment in cash. The scheme has also incentivized the involvement of private GPs in managing chronic diseases.

In recent years, the Ministry of Health has also launched the Community Health Assist Scheme (CHAS), a scheme that enables Singapore Citizens from lower- to middle-income households to receive subsidies for medical and dental care at participating GP and dental clinics near their homes.

\section{Hopes and aspirations}

\section{Interface between general practice, community-based programs and secondary/tertiary services}

More than ten years ago, Singapore realized that the traditional tiers of primary, secondary, tertiary and quaternary care centers were too rigid for effective chronic disease management. Two terms have been bandied about: right-siting and integrated care. Integrated care refers to the organizational process of coordination which seeks to achieve seamless and continuous care, tailored to the patient's needs and based on a holistic view of the patient. ${ }^{5}$ True integration of care often requires a painful demolition of old rigid structures, working across traditional health service boundaries. Functional integration needs information technology support, clinical integration needs distinct work processes, and virtual integration needs a coordinating body such as a Chronic Disease Management Office to orchestrate patient and information transition across different care settings as well as the monitoring of clinical quality indicators (Figure 2).

In 2004, the concept of right-siting was first used to describe the principle that chronic disease patients should be managed in primary care instead of specialist settings. In 2005, the National Healthcare Group developed the Chronic Disease Management System (CDMS), a region-wide disease register that synchronizes clinical health records. ${ }^{6}$ In the same year, the Singapore Health Services (SingHealth) regional cluster initiated the Delivering On Target (DOT) Programme, leading to positive health outcomes in diabetic patients. The aim of the DOT Programme was to right-site chronic patients to primary care, benchmarked against evidence-based best practice clinical guidelines. It expanded into a nation-wide program in 2008.

In July 2011, the National University Hospital (NUH) and Frontier Healthcare, an eight-clinic private GP chain, announced a collaboration to fine-tune the shared care model. Stable diabetes patients are referred to Frontier clinics by NUH doctors for co-management. Patients under the program are assured of the same level of care when they consult the family physicians at Frontier clinics. 
The family physicians will follow the same set of care components for diabetic patients practised by the specialists at NUH. The partnership aims to ensure patients receive timely care for their conditions at greater convenience, at clinics nearer their homes.

In 2014, in line with the government's Primary Care Master Plan which promotes the participation of public and private sectors in providing comprehensive and integrated care to patients with stable chronic conditions, SingHealth set up a similar service with a Family Medicine Clinic at Chinatown Point.

\section{Facilitating multi-disciplinary care}

The first diabetes center for one-stop patient care was established in 1990 in Singapore General Hospital (SGH). Since then, every restructured hospital/regional healthcare system has established a multi-disciplinary diabetes center. In the primary healthcare sector, government polyclinics also have on-site foot and retinal screening and access to diabetes nurse educators and dieticians.

The co-location of outpatient services with interests in diabetes management (endocrinology, renal medicine, internal medicine, vascular surgery and podiatry from SGH and vitreo-retinal service from Singapore National Eye Centre) in 2015 provides an opportunity to establish a new diabetes model of care under a unifying umbrella of the Singhealth-Duke Diabetes Centre. We are also working to streamline clinical services across the continuum of diabetes care including pediatric and adolescent care, dental services and cardiology/ cardiothoracic care.

\section{Improve accessibility and quality of diabetes self- management education}

We have moved to broaden delivery of care by expanding the healthcare team to include several types of healthcare professionals. Team care can minimize patients' health risks by assessment, intervention, and surveillance to identify problems early and initiate timely treatment.

One area for improvement is the use of effective behavioral interventions to lower the risk of diabetes and improve glycemic control and cardiovascular risk profiles. Patients' participation in treatment decisions, personal selection of behavioral goals, patient education and training, and active self-management can all improve diabetes control. This, in turn, can lead to increased patient satisfaction with care, better quality of life, improved health outcomes, and ultimately, lower healthcare costs. We recognize that there is plenty to do in this arena. We were glad to include a new chapter addressing psychosocial needs and selfmanagement in the most recent edition of our local Clinical Practice Guidelines for diabetes mellitus. ${ }^{7}$

\section{The National Electronic Health Record (NEHR)}

The NEHR was operationalized in the end or 2011, to integrate and share medical records nationwide to support the seamless delivery of patient care. Patients served by the public sector healthcare institutions already have a single health record shared among our institutions, such as hospitals, specialist outpatient clinics and polyclinics. All community hospitals, 56 community healthcare providers and close to $40 \%$ of GP clinics have access to NEHR. The Ministry of Health is actively working with the remaining private healthcare providers not yet on board to encourage their participation.

The NEHR will continue to develop new IT functions and provide information to support the integration of care services for the patient across the healthcare sector. This includes the development of the Continuity of Care Record (CCR) functionality which would provide a mechanism for institutions to share the patient's active problem list and care plan, with the end state being a seamless integration with hospital electronic medical record (EMR) systems. Future developments will also include the use of data analytics to support both point-ofcare decision making and national planning for the Ministry of Health.

The Health IT Master Plan (HITMAP) was developed in 2015 with public and private sector stakeholders to anticipate and meet emerging challenges and growing healthcare needs.

\section{Harnessing technology}

Another objective of the Singapore Diabetes care model is the use of technology to improve service coordination for better outcomes and more efficient care. An excellent example can be seen in the retinopathy screening services. Diabetic retinopathy is the leading cause of new blindness in working adults in developed countries, including Singapore. Before the advent of a national screening program, patients in the primary care sector had to travel physically to designated polyclinics or centers for an appointment. Retinal images were interpreted by family physicians housed within the polyclinics, with a turnaround time of two to four weeks for grading. Inherent weaknesses with this system were inconsistencies in the grading outcomes, cumbersome quality assurance maintenance, and poor cost-effectiveness especially in terms of manpower costs. ${ }^{8}$

The Singapore Integrated Diabetic Retinopathy Programme (SiDRP) was thus conceptualized and launched in 2010, based on telemedicine and centralized reading centers. All retinal images captured are routed electronically to the Singapore Eye Research Institute (SERI) ocular reading centre for same-day reading and reporting. A remarkable 90 to $95 \%$ of images are read and reported in a one-hour time frame. Appropriate referrals are made to ophthalmologists for further assessment and treatment, if necessary, for the same visit at the polyclinic. Patient accessibility has been greatly increased, with image capture sites established beyond conventional touchpoints like polyclinics, to include mobile screening 
vans and optometrists. With these patient volumes, there is also the opportunity to generate a powerful cohort of patients with retinal phenotyping for outcome studies.

\section{CONCLUSION}

The main aim of the Singapore model is to configure a system that is efficient and cost-effective, in order to prevent and delay the onset of diabetes and diabetesrelated complications, and to improve the quality of life of people who have diabetes.

This is done through a mixed financing system and a mixed delivery model, underpinned by dual principles of affordability and individual responsibility. Any model of care, not just Singapore's, involves prescribers, patients, regulators, influencers and payers. There is already a conscious effort to structure our decision trees and algorithms on evidence-based medicine drawn from an Asian population. However, there is a paucity of realworld data to help make effective decisions on the value propositions of diabetes therapies that are currently available.

Integration of care for patients with diabetes is a noble and worthwhile aspiration. We must remember that integration is a means to an end, and not an end in itself. We need flexible, networked solutions to bring about positive change, especially in the areas of GP-coordinated prevention programs, ready and targeted availability of new technology in diabetes diagnostics and therapeutics, and patient self-management and self-care.

\section{References}

1. Prime Minister's Office Singapore. Transcript of speech by Prime Minister Lee Hsien Loong at Universal Health Coverage Ministerial Meeting, Grand Copthorne Waterfront. Web. 10 Aug. 2015. http://www.pmo.gov.sg/mediacentre/transcript-speech-prime-ministerlee-hsien-loong-universal-health-coverage-ministerial.

2. Ministry Of Health Singapore. Singapore Burden of Disease Study 2010. Web. 11 Aug.2015. https://www.moh.gov.sg/content/ moh_web/home/publications/reports/2014/singapore-burden-of-diseasestudy- 2010.html.

3. Phan TP, Alkema L, Tai ES, Tan KH, Yang Q, Lim W-Y, et al. Forecasting the burden of type 2 diabetes in Singapore using a demographic epidemiological model of Singapore. BMJ Open Diabetes Res Care. 2014;2(1):e000012. http://dx.doi.org/ 10.1136/bmjdrc-2013-000012.

4. Ministry Of Health Singapore. Costs and Financing. Web. 10 Aug. 2015. https://www.moh.gov.sg/content/moh_web/home/costs_and_ financing.html.

5. Mur-Veeman I, Hardy B, Steenbergen M, Wistow G. Development of integrated care in England and the Netherlands. Health Policy. 2003;65(3):227-241. http://dx.doi.org/10.1016/S0168-8510(02)00215-4.

6. Ang YG, Wu CX, Toh MPHS, Chia KS, Heng BH. Progression rate of newly diagnosed impaired fasting glycemia to type 2 diabetes mellitus: A study using the National Healthcare Group Diabetes Registry in Singapore. J Diabetes. 2012;4(2):159-163. http://dx.doi.org/ 10.1111/j.1753-0407.2011.00169.x.

7. Goh SY, Ang SB, Bee YM, Chen RYT, Gardner DS, et al. Ministry of health clinical practice guidelines: Diabetes mellitus. Singapore Med J Singapore M J. 2014;55(6):334-47. http://dx.doi.org/10.11622/ smedj.2014079.

8. Bhargava M, Cheung CY, Sabanayagam C, Kawasaki R, Harper CA, Lamoureux EL, et al. Accuracy of diabetic retinopathy screening by trained non-physician graders using non-mydriatic fundus camera. Singapore Med J. 2012 Nov;53(11):715-9.

Articles and any other material published in the JAFES represent the work of the author(s) and should not be construed to reflect the opinions of the Editors or the Publisher. Authors are required to accomplish, sign and submit scanned copies of the JAFES Declaration: that the article represents original material, that is not being considered for publication or has not been published or accepted for publication elsewhere. Consent forms, as appropriate, have been secured for the publication of information about patients; otherwise, authors declared that all means have been exhausted for securing such consent. The authors have signed disclosures that there are no financial or other relationships that might lead to a conflict of interest. All authors are required to submit Authorship Certifications that the manuscript has been read and approved by all authors, and that the requirements for authorship have been met by each author.

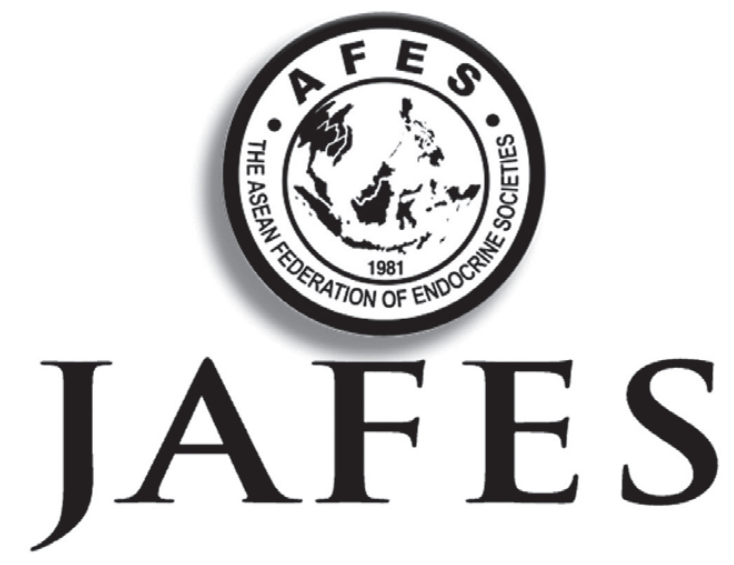

\section{Had an invigorating discussion in Grand Rounds? Share your Clinical Case Seminars at JAFES@Asia.com.}

